\title{
Microemulsions with Nonionic Surfactants and Mint Oil
}

\author{
Monzer Fanun* \\ Colloids and Surfaces Research Laboratory, Faculty of Science and Technology, Al-Quds University, 51000 East \\ Jerusalem, Palestine
}

\begin{abstract}
A combination of electrical conductivity, dynamic viscosity, dynamic light scattering and small angle X-ray scattering methods was used to study the properties of the water + propylene glycol/mixed nonionic surfactants/ peppermint oil + ethanol U-type microemulsions which can be diluted with any amount of water. The surfactants were sucrose laurate and ethoxylated mono-di-glyceride. The mixing ratios $(\mathrm{w} / \mathrm{w})$ of the mixed surfactants and peppermint oil/ethanol equal unity and that of water/propylene glycol equals to two. The electrical conductivity and periodicity of the microemulsions increases with the aqueous phase content while the dynamic viscosity decreases. The variations in the values of the correlation length with the aqueous phase contents indicate the onset of structural transitions. Structural transitions from the water-in-oil to a bicontinuous phase then inversion to oil-in-water occurs in the system.
\end{abstract}

Keywords: Phase behavior, hydrodynamic radius, periodicity, correlation length, structural transitions.

\section{INTRODUCTION}

Microemulsions are transparent and thermodynamically stable mixtures of two immiscible liquids, i.e., water and oil, brought together by means of an appropriate surfactant or surfactant/co-surfactant mixture [1]. Microemulsions, based on nonionic surfactants, have been extensively investigated, both from a formulation, and a structural point of view [1-8]. In almost all nonionic microemulsions the nonionic surfactants used are single surfactants. However, mixed nonionic surfactants are also used [1, 9-14]. Mixing ethoxylated surfactants and polyol type nonionic surfactants such as sucrose esters of fatty acids is promising for the formulation of biocompatible microemulsions. Sucrose laurate (L1695) and ethoxylated mono-di-glyceride (EMDG) are nonionic biodegradable surfactants formed from renewable sources. The physical properties of sucrose esters are somewhat unique. Unlike the ethoxylated surfactants, temperature-insensitive sucrose-ester-based microemulsions are described in the literature [15-17]. It was also shown that mixing sucrose laurate with ethoxylated mono-di-glyceride tuned the behaviour of the later and microemulsions systems with improved properties were formed [14, 18-24]. A few numbers of authors [25-27] reported the use of edible oils other than triglycerides for the formation of food, cosmetic, or pharmaceutical grade microemulsions. The placement of the solubilized oil in the surfactant aggregates highly affects the change in surfactant layer curvature. In previous studies [1924], we reported on microemulsions formulation using cyclic hydrocarbons. These oils influence the surfactant layer curvature in ag gregates or self organized structures when solubilized. It is known that cyclic hydrocarbons tend to penetrate in the surfactant layer and widen the effective cross-sectional area per surfactant. As a result, the surfactant

*Address correspondence to this author at the Colloids and Surfaces Research Laboratory, Faculty of Science and Technology, Al-Quds University, 51000 East Jerusalem, Palestine; Tel.: + 970227997 53; Fax: + 970 227969 60; E-mail: fanunm@gmail.com,mfanun@ science.alquds.edu layer curvature becomes less positive or negative. In the present work, the structural properties of mixed sucrose laurate and ethoxylated mono-di-glyceride and peppermint oil microemulsions will be investigated in more detail by means of electrical conductivity, dynamic viscosity, dynamic light scattering (DLS) and small-angle X-ray scattering (SAXS), These techniques can be used to obtain valuable information on size, shape, internal structure, and diffusion dynamics of colloids and complex fluids. Especially their use for the characterization of self-organizing amphiphilic systems like microemulsions has proven to be very effective [28].

\section{EXPERIMENTAL}

\subsection{Materials}

The sucrose laurate (L1695) (Fig. 1A) was obtained from Mitsubishi-Kasei Food Corp., (Mie, Japan). The purity of combined Lauric acid equals $95 \%$, the esters compositions are $80 \%$ monoester and $20 \% \mathrm{di}$, tri and polyester, HLB equals 16. Ethoxylated mono-di-glyceride (EMDG) (MAZOL 80 MG KOSHER) was obtained from BASF Corporation (Gurnee, Illinois, USA). Ethoxylated mono-di-glyceride is composed of a mixture of stearate and palmitate partial esters of glycerin ethoxylated with approximately 20 moles of ethylene oxide per mole of alpha-mono-glyceride reaction mixture with HLB equals 13.5 (Fig. 1B). Peppermint oil (98\%) and 1, 2-Propandiol (Propylene glycol, PG) ( $\geq 99.5 \%)$ were purchased from BDH (Poole, UK). Ethanol (minimum 99.8\%) was purchased from Sigma Chemicals Co. (st. Louis, USA). All of the components were used as supplied without further purification. Triple distilled water was used.

\subsection{Methods}

\subsubsection{Pseudoternary Phase Diagrams at Constant Tem- perature}

The phase behavior of a system consisting of water, oil, mixed surfactants, and cosurfactants may be described on 
a phase diagram whose apexes respectively represent the pure components. However, it is more convenient to describe the phase behavior on a pseudo-ternary phase triangles. Obviously, a fixed (weight, volume or mole) ratio must be chosen for any two of the components and one of the triangle vertices represents $100 \%$ of this binary mixture. Mixtures at fixed weight ratios of oil, surfactant and ethanol were prepared in culture tubes sealed with Viton lined screw caps. Water and propylene glycol were then added dropwise until its solubilization limit was reached. After this point, larger increments of aqueous phase were added. Vigorous stirring followed all of the aqueous phase additions on a vortex mixer. The time for equilibration between additions of successive aliquots was typically, from a few minutes up to 24 hours. Phase transitions detected visually by the appearance of cloudiness or sharply defined separated phases. The completion of this process was hastened by centrifuging the samples. The phase diagrams were determined at $25 \pm 0.5^{\circ} \mathrm{C}$.

\section{$\mathrm{CH}_{3}\left(\mathrm{CH}_{2}\right)_{10} \mathrm{COOCH}_{2}$}

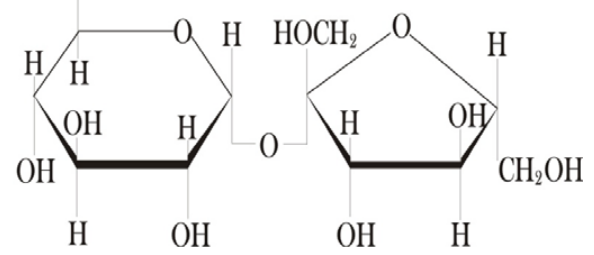

(A)

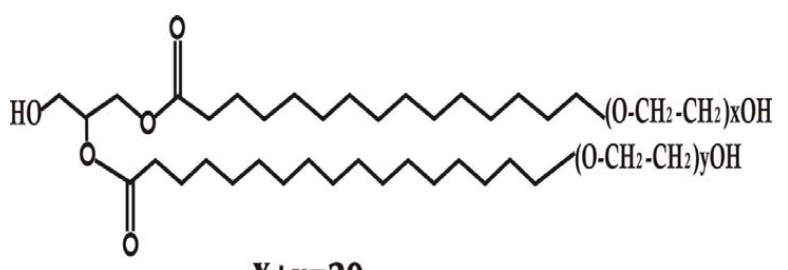

$X+y=20$

(B)

Fig. (1). Chemical structures of [A] sucrose laurate, and [B] ethoxylated mono-di-glyceride.

\subsubsection{Electrical Conductivity Measurements}

Conductivity measurements were performed at $25 \pm 0.5^{\circ} \mathrm{C}$ on samples the compositions of which lie along the one phase channel, using conductivity meter, the conductivity cell used is Tetra Con ${ }^{\circledR} 325$, the electrode material is graphite and the cell constant is $0.475 \mathrm{~cm}^{-1} \pm 1.5 \%$. The range of application is between $1 \mu \mathrm{S} / \mathrm{cm}$ to $2 \mathrm{~S} / \mathrm{cm}$ with an accuracy of $\pm 0.5 \%$, and the temperature range is from -5 to $100{ }^{\circ} \mathrm{C}$. The electrode was dipped in the microemulsion sample until equilibrium was reached and reading becomes stable. Reproducibility was checked for certain samples and no significant differences where observed. The constant of the conductivity cell was calibrated using standard $\mathrm{KCl}$ solutions and checked a minimum of three times during the course of the working shift.

\subsubsection{Viscosity Measurements}

Viscosity was measured using a rotational viscometer, model DV-1PL spindle from Anton Paar GmbH (Graz, Austria). "Double cylinder" geometry was used. Viscosities at $200 \mathrm{~s}^{-1}$ shear rate were obtained at $25 \pm 0.5^{\circ} \mathrm{C}$. Reproducibility (triplicate) was checked for the samples and no significant differences $( \pm \mathrm{SD})$ where observed.

\subsubsection{Dynamic Light Scattering (DLS)}

Microemulsions samples were inserted into a $10 \mathrm{~mm}$ diameter borosilicate test tube and centrifuged for $15 \mathrm{~min}$ at $3000 \mathrm{rpm}$ in order to remove dust. The test tube was then placed in a temperature-controlled vat of toluene as the index matching fluid. The light source was an argon ion laser (Spectra Physics- Lexel; $\lambda=514.5 \mathrm{~nm}$ ). Photons scattered by the sample were collected by an ITT PW130 photomultiplier tube mounted at $90^{\circ}$ to the incident laser beam. The photoelectron count-time autocorrelation function was calculated with a BI 2030 AT (Brookhaven Instruments) digital autocorrelator and was analyzed using the constrained regularization algorithm, CONTIN [29, 30] to give an intensity weighted distribution of the translational diffusion coefficients $D_{z}$. Particle hydrodynamic size was calculated from the translational diffusion coefficient using the StokesEinstein relationship:

$\mathrm{D}_{\mathrm{H}}=\mathrm{k}_{\mathrm{B}} \mathrm{T} / 6 \pi \eta \mathrm{D}_{\mathrm{z}}$

where $\mathrm{D}_{\mathrm{H}}$ is the hydrodynamic diameter, $\mathrm{k}_{\mathrm{B}}$ is Boltzmann's constant, $\mathrm{T}$ is the absolute temperature and $\eta$ is the solvent viscosity. The results are averages of 3 or 4 experiments. $D_{H}$ is obtained from the positions of the peaks of the intensity weighted size distribution function.

\subsubsection{Small Angle X-ray Scattering (SAXS)}

Scattering experiments were performed using $\mathrm{Ni}$-filtered $\mathrm{Cu} \mathrm{K} \alpha$ radiation $(0.154 \mathrm{~nm})$ from a Eliott GX6 rotating $\mathrm{X}$-ray generator that operated at a power rating up to $1.2 \mathrm{~kW}$. $\mathrm{X}$-radiation was further monochromated and collimated by a single Franks mirror and a series of slits and height limits and measured by a linear position-sensitive detector. The sample was inserted into 1-1.5 mm quartz or lithium glass capillaries which were then flame-sealed. Each sample was checked before and after the experiment to verify that no fluid had been lost during the time of exposure, approximately $3 \mathrm{~h}$. The temperature was maintained at $25 \pm 1^{\circ} \mathrm{C}$. The sample-to-detector distance was $0.46 \mathrm{~m}$, and the scattering patterns were measured using the Lake procedure [31].

\subsubsection{X-ray Data Analysis}

In this case, the scattering patterns after background subtraction were fit to the expression due to Teubner and Strey [32]:

$I(q)=\left(1 / a_{2}+c_{1} q^{2}+c_{2} q^{4}\right)+b$

Where $\mathrm{I}(\mathrm{q})$ is static scattering density distribution with the constants $\mathrm{a}_{2}, \mathrm{c}_{1}, \mathrm{c}_{2}$ and $\mathrm{b}$ obtained by using the Levenburg-Marquardt procedure [33]. Such a functional form is simple and convenient for the fitting of spectra. Equation 3 corresponds to a real space correlation function of the form

$\gamma(\mathrm{r})=(\sin \mathrm{kr} / \mathrm{kr}) \mathrm{e}^{-\mathrm{r} / \mathrm{\xi}}$

The correlation function $\gamma(\mathrm{r})$ describes a structure with periodicity $\mathrm{d}=(2 \pi / \mathrm{k})$ damped as a function of correlation length $\xi$. This formalism also predicts the surface to volume ratio, but because this ratio is inversely related to the correla- 
tion length and therefore must go to zero for a perfectly ordered system, calculated values are frequently found to be too low [34]. d and $\xi$ are related to the constants in Equation (2) by Teubner and Strey [32]:

$$
\begin{aligned}
& \mathrm{d}=2 \pi\left[(1 / 2)\left(\left(\mathrm{a}_{2} / \mathrm{c}_{2}\right)\right)^{1 / 2}-\left(\mathrm{c}_{1} / 4 \mathrm{c}_{2}\right)\right]^{-1 / 2} \\
& \xi=\left[(1 / 2)\left(\left(\mathrm{a}_{2} / \mathrm{c}_{2}\right)\right)^{1 / 2}+\left(\mathrm{c}_{1} / 4 \mathrm{c}_{2}\right)\right]^{-1 / 2}
\end{aligned}
$$

\section{RESULTS AND DISCUSSION}

\subsection{Phase Behavior}

The pseudoternary phase diagrams of the water + propylene glycol/sucrose monolaurate/ ethoxylated monodi-glyceride/peppermint oil+ ethanol system was studied at $25^{\circ} \mathrm{C}$. The mixing ratios (w/w) of sucrose monolaurate/ ethoxylated mono-di-glyceride and ethanol/oil equal unity. The water/propylene glycol mixing ratio $(\mathrm{w} / \mathrm{w})$ equals 2 . Fig. (2) presents the phase behavior of an isotropic and low-viscosity one-phase microemulsion region designated by $(1 \phi)$ is present; the remainder of the phase diagram represents a two phase region composed of water continuous micellar solution with excess oil designated by heterogeneous region. The maximum water solubilization achieved using a mixture of nonionic surfactants could be due to two different synergism mechanisms. The first, the maximum water solubilization capacity could be related to simple additive contributions of the surfactant material in the HLB 9-13 region with no apparent additional benefit from synergism between the two surfactant. Secondly, synergistic effect must cause the majority of the two surfactants to preferentially partition at the interface that allows larger interfacial area and thus high levels of solubilization. When the microemulsions were composed of equal amounts of surfactants, the synergistic effect found to be more pronounced. This important change in the water solubilization is achieved at the ethoxylated mono-di-glyceride to sucrose laurate molar ratio of 1 to 3 .

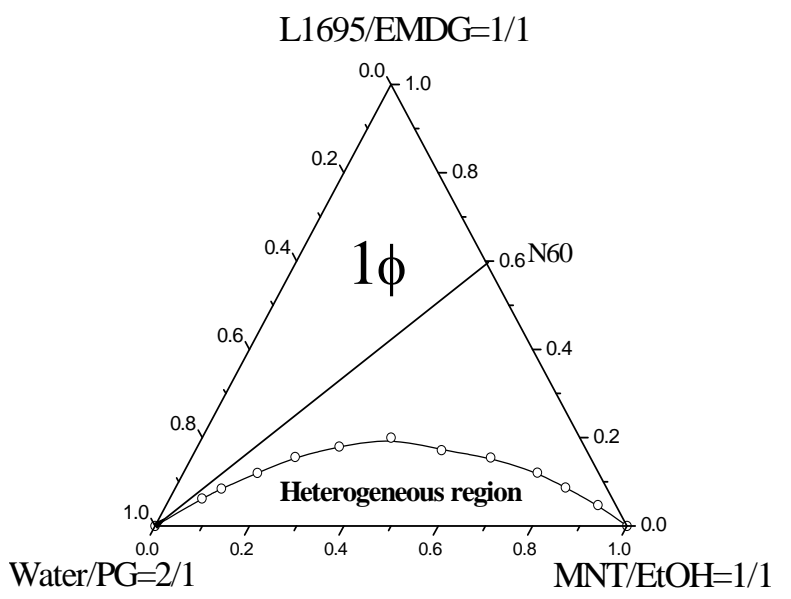

Fig. (2). Pseudoternary phase behavior of water / propylene glycol/ sucrose laurate / ethoxylated mono-di-glyceride/ peppermint oil /ethanol system at $25^{\circ} \mathrm{C}$. The mixing ratios $(\mathrm{w} / \mathrm{w})$ of sucrose laurate/ ethoxylated mono-di-glyceride and ethanol/oil equal unity and that of water/propylene glycol equals 2 . The one phase region is designated by $1 \phi$, and the multiphase region is designated as heterogeneous region. N60 is the water dilution line where the weight ratios of sucrose laurate/ ethoxylated mono-di-glyceride /peppermint oil/ ethanol equal to $3 / 3 / 2 / 2$.

\subsection{Transport Properties}

\subsubsection{Electrical Conductivity}

The present microemulsion system, containing mixed nonionic surfactants, show signs of electrical conductivity in spite of its nonionic type. A possible explanation for such behavior is the natural origin of the surfactants, which is associated with the presence of impurities that contribute to the electrical conductance of the systems studied. The variation on the electrical conductivity of the microemulsions upon dilution with aqueous phase was determined along the water dilution line N60 is shown in Fig. (3). The electrical conductivities increase with the increase in the aqueous phase volume fraction. The conductivity profile reveals the existence of four regions manifested in different slopes. The

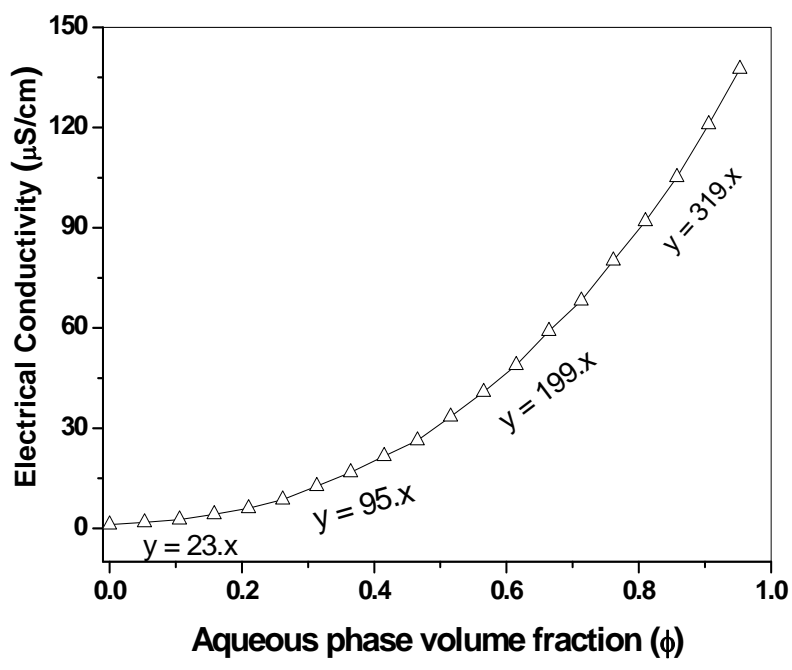

Fig. (3). Electrical conductivity $(\sigma)$ as function of aqueous phase volume fraction along the N60 dilution line of the system where the phase diagram is presented in Fig. (2).

first region is from 0.01 to 0.25 aqueous phase volume fractions and its slope equation is $y=23 . x$, while in the region of $0.25-0.52$ aqueous phase volume fractions, the slope equation is more gradual y $=95 . x$. In the third region aqueous phase volume fractions between 0.52-0.8, the slope equation is $y=199 . x$. In the fourth region, the slope is the steeper and equals to $y=319$.x. In our previous studies [20, 21, 35, 36], we learned that in the U-type systems the reverse micelles that are formed in the oil phase slowly swell and distort upon addition of aqueous phase, and at a certain point the water migrates out of the inner phase and sponge-like domains are formed termed the bicontinuous phase. Upon further dilution, full inversion occurs and the water captured within the oil becomes a continuous phase. Structural transition, from the water-in-oil to a bicontinuous phase, occurs in the system at ca. $0.25-0.30$ aqueous phase volume fractions. In the first structural transition appears the water cores are evenly distorted and upon adding steadily more water, they no longer reveal spherical or disc-like shapes but turn into "worm-like" water domains that are dispersed into the oil continuous domains. At certain aqueous phase content, the water phase begins to drift to the outer phase, the curvatures adjust, and the conductivity increases. The bicontinuous region is quite large and exists within 0.25 to 0.75 aqueous phase volume 
fractions where the water and the oil phases are interwoven. The other transition occurs between 0.75 and 0.80 aqueous phase volume fractions and is difficult to detect since the process is quite gradual. This slope deflection is a sign of the complete change of the bicontinuous structure into oil-in-water droplets, and the water becomes the continuous phase.

\subsubsection{Dynamic Viscosity}

Dynamic viscosity in depends mainly on the microemulsion type, i.e., the microstructure and shape of aggregates, and interactions between dispersed droplets. Therefore, it can be used to acquire important information concerning structural transformations in microemulsions, although not necessarily the points at which the transition occurs or is completed $[36,37]$. The variation in dynamic viscosity as a function of aqueous phase volume fraction is shown in Fig. (4). In the present study, the transition point, observed in the viscosity data, match with the transition point determined by electrical conductivity measurements. Dynamic viscosity measurements suggest transformation of the system structure from water-in-oil microemulsions for water volume fractions below 0.20 , via bicontinuous between 0.20 to 0.52 water volume fractions and then to inverted bicontinuous region between 0.52 to 0.80 water volume fractions, and then to oilin-water microemulsions for water volume fraction above 0.80 . It should be noted, that while the electrical conductivity reflects structural transitions, the viscosity reflects mostly structural interaction variations and is indicative of the sharp collapse of the oil-in-water bicontinuous network. In the first region (i.e. water volume fractions below 0.2 ), the interconnected water and oil "channels" increase structural interactions progressively and therefore viscosity. However, with further dilution, the bicontinuous structure gradually disintegrates, and transition into oil-in-water microemulsion occurs, which is reflected in a sharp decrease in the viscosity. In this region, the head groups' hydration is strongly increased and the packing parameter drops to values less than one third. The viscosity continues to decrease as the nanodroplets decrease in number and size, and become more and more diluted with aqueous phase. The progressive dilution decreases the interdroplet interactions.

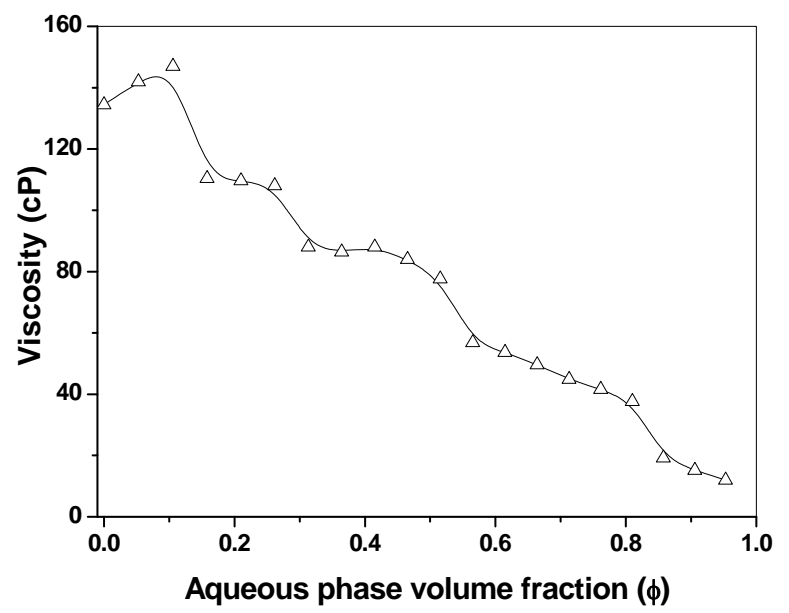

Fig. (4). Dynamic viscosity $(\mu)$ as function of aqueous phase volume fraction along the N60 dilution line of the system where the phase diagram is presented in Fig. (2).

\subsection{Diffusion Properties}

Dynamic light scattering (DLS) is a rapid method used to acquire information on the size of the particles in relatively simple and dilute systems. In this section, we used the DLS technique to investigate the microemulsion systems, as a function of temperature at aqueous phase volume fraction equals 0.91 along the dilution line N60. The values of the hydrodynamic radius $\left(R_{H}\right)$ as function of temperature in the aqueous phase rich region are presented in Table $1 . R_{H}$ increases with temperature increase indicating that the tails of the surfactants molecules residing on different droplets or fusion of droplets which lowers the curvature energy makes the micelles grow in size as the temperature increases The microemulsion droplets deform by thermal fluctuations. The droplets may undergo attractive interactions that lead to aggregation between the droplets. Similar results of the behavior of the hydrodynamic radius as function of temperature were reported in our previous studies [14, 18, 20, 38].

Table 1. Values of the Hydrodynamic Radius $\left(\mathbf{R}_{H}\right)$ for the Water + Propylene Glycol/Sucrose Laurate/ Ethoxylated Mono-Di-Glyceride/ Peppermint Oil + Ethanol System Along the N60 Dilution Line at $25^{\circ} \mathrm{C}$. The Aqueous Phase Volume Fraction Equals 0.9. The Mixing Ratios (w/w) of Sucrose Laurate/ Ethoxylated Mono-Di-Glyceride and that of Ethanol/ Peppermint Oil Equal Unity and that of Water/ Propylene Glycol Equals Twice. Values of $\mathbf{R}_{\mathbf{H}}$ are Calculated from Equation (1)

\begin{tabular}{|c|c|}
\hline \multirow{2}{*}{ Temperature (K) } & Hydrodynamic Radius $\mathbf{R}_{\mathbf{H}}(\mathbf{n m})$ \\
\cline { 2 - 2 } & Free \\
\hline \hline 293 & 9 \\
\hline 298 & 11 \\
\hline
\end{tabular}

\subsection{Microstructure Properties}

Small angle X-ray scattering (SAXS), probe the pertinent colloidal length scales of $1-100 \mathrm{~nm}$ and therefore is the method of choice for determining the size, shape, and internal structure of colloidal particles. The scattering intensity depends on the different scattering length densities of the particles and the solvent. For SAXS the scattering length density is proportional to the electron density, which is a linear function of the number of electrons. Consequently, SAXS is particularly useful for investigating the interfacial region of aggregates composed of surfactant molecules [39]. However, at the time of these experiments, SAXS data were not measured on absolute scale and so all data are presented in arbitrary units. SAXS was used to obtain information on the size of the microemulsions and on their internal structure. Only the polar headgroups and water regions are visible with SAXS experiments due to the fact that their electron densities are higher than the electron density of the surrounding oil. SAXS measurements were used to study the microstructure parameters (i.e. periodicity, and correlation length) of the microemulsions. Fig. (5) presents the Characteristic profiles as an example of what happens in the microemulsions as a function of the water volume fraction $(\phi)$ along the dilution line N60. According to the Teubner-Strey equation [32] 
(Equation 2) we were able to derive from values of the periodicity, d, and correlation length, $\xi$, as described in the experimental section (Equations 3-5). The periodicity (d) calculated by Teubner and Strey's model [32] was plotted against the water volume fraction as shown in Fig (6). The $\mathrm{d}$ values increase as the water content increases. Different regions are observed in this curve corresponding to the presence of structural transitions along the dilution line studied from water-in-oil to bicontinuous to oil-in-water microemulsions. Fig. (7) presents the correlation length $(\xi)$ of the microemulsions. $\xi$, reflects the degree of order in the microemulsion. Different regions are also observed in this curve corresponding to the presence of structural transitions. Three maxima are observed in the correlation length as function of the aqueous phase volume fraction. These maxima correspond to the loci of structural transitions. The loci correspond largely to the transition points observed by the measurements of both the electrical conductivity and the dynamic viscosity. Fig. (8) shows a schematic presentation of the suggested structural transitions along the N60 dilution line.

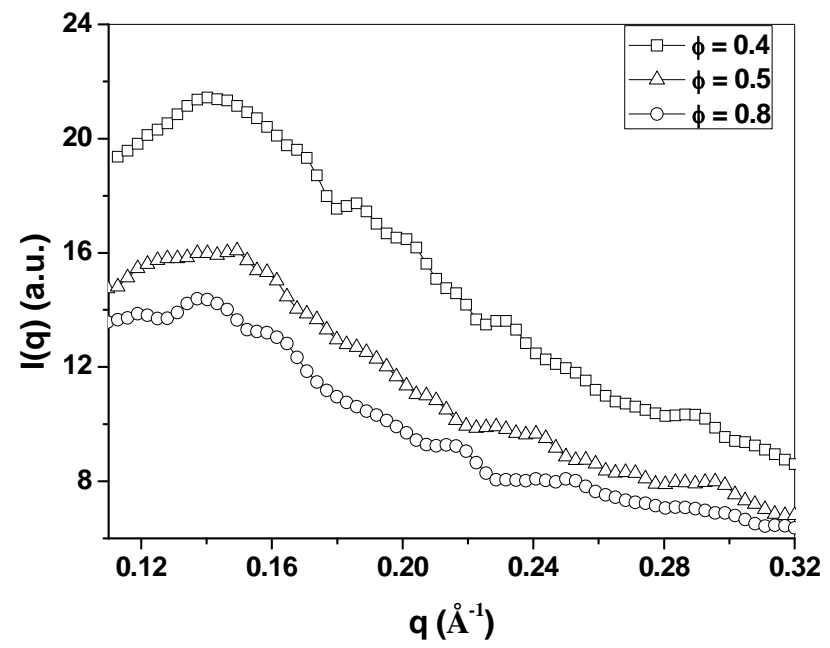

Fig. (5). Small angle X-ray scattering curves for samples whose compositions lie along the dilution line N60 as function of aqueous phase content along the dilution line N60 of the system where the phase diagram is presented in Fig. (2).

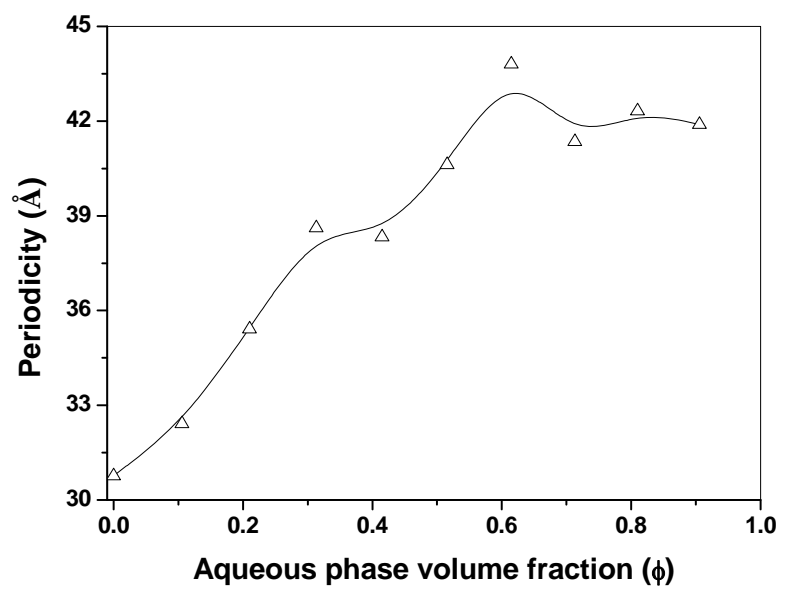

Fig. (6). Periodicity (d) of the microemulsions samples as function of aqueous phase content along the dilution line N60 for the systems presented in Fig. (5).

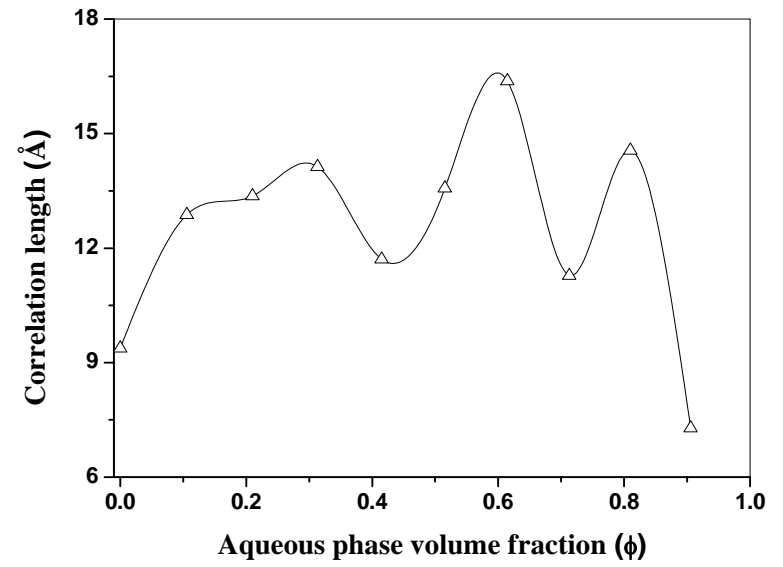

Fig. (7). Correlation length, ( $\xi$ ) of the microemulsions samples as function of aqueous phase content along the dilution line N60 for the systems presented in Fig. (5).

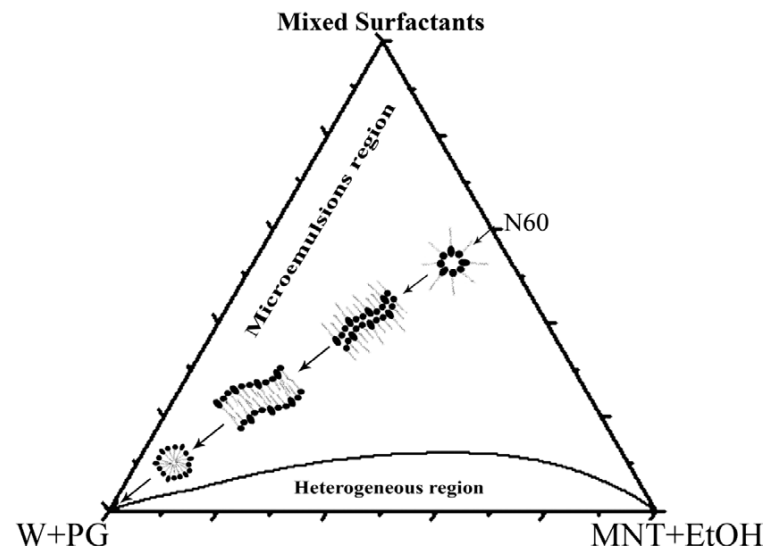

Fig. (8). Schematic presentation (not for scale) of the structural transitions along the N60 dilution line in the system where the phase diagram is presented in Fig. (2).

\section{CONCLUSIONS}

We were able to formulate and characterize biocompatible microemulsions that can be diluted with any amount of water or oil. These microemulsions form channels upon addition of water that leads to the formation of bicontinuous microstructures. The microstructure parameters of these systems change indicating structural transitions with the increase in the aqueous phase content. The variation in the structural and transport parameters lead to the conclusion that in the one phase microemulsions region the water-in-oil to bicontinuous to oil-in-water droplets transitions occur with the aqueous phase content. The formulation and characterization of these systems gives new insight on the behavior of new types of both safe surfactants and oil.

\section{REFERENCES}

[1] Fanun, M. Microemulsions: Properties and Application. Taylor and Francis/CRC Press: Boca Raton, USA, 2009.

[2] Strey, R.; Jonstromer, M. Role of medium-chain alcohols in interfacial films of nonionic microemulsions. J. Phys. Chem., 1992, 96, 4537-4542. 
[3] Bansal, V. K.; Shah, D. O.; O'Connell, J. P. Influence of alkyl chain length compatibility on microemulsion structure and solubilization. J. Colloid Interface Sci., 1980, 75, 462-475.

[4] Shinoda, K.; Shibata, Y. Principles for the attainment of minimum oil-water interfacial tension by surfactants: The characteristics of organized surfactant phase. Colloids Surf., 1986, 19, 185-196.

[5] Kahlweit, M.; Strey, R.; Busse, J. Microemulsions: A qualitative thermodynamic approach. J. Phys. Chem., 1990, 94, 3881-3894.

[6] Paul, B. K.; Moulik, S. K. Microemulsions: An overview. J. Dispers. Sci. Technol., 1997, 18, 301-367.

[7] Kunieda, H.; Yamagata, M. Mixing of nonionic surfactants at water-oil interfaces in microemulsions. Langmuir, 1993, 9, 33453351.

[8] Garti, N.; Aserin, A.; Ezrahi, S.; Wachtel, E. Water Behavior in Nonionic Surfactant Systems I: Subzero Temperature Behavior of Water in Nonionic Microemulsions Studied by DSC. J. Colloid Interface Sci., 1996, 178, 60-68.

[9] Kunieda, H.; Solans, C. Industrial Applications of Microemulsions, Marcel Dekker, Inc., New York, 1996.

[10] Scamehorn, J.F. Phenomena in Mixed Surfactant Systems; American Chemical Society, Washington DC, 1986.

[11] Holland, P.M.; Rubingh D.N. Mixed Surfactant Systems, American Chemical Society, Washington, DC, 1992.

[12] Ogino, K.; Abe, M. Mixed Surfactant Systems, Surfactant Science Series 46, eds.; Marcel Dekker, Inc. New York, 1992.

[13] Huibers, P.D.T.; Shah, D.O. Evidence for synergism in nonionic surfactant mixtures: enhancement of solubilization in Water-in-Oil microemulsions. Langmuir, 1997, 13, 5762-5765.

[14] Fanun, M.; Salah, Al-Diyn, W. Electrical conductivity and self diffusion-NMR studies of the system: Water/sucrose laurate/ethoxylated mono-di-glyceride/isopropylmyristate. Colloids Surf. A, 2006, 277, 83-89.

[15] Kunieda, H.; Ushio, N.; Nakano, A.; Miura, M. Three-Phase Behavior in a Mixed Sucrose Alkanoate and Polyethyleneglycol Alkyl Ether System. J. Colloid Interface Sci., 1993, 159, 37-44.

[16] Aramaki, K.; Kunieda, H.; Ishitobi, M.; Tagawa, T. Effect of added salt on three-phase behavior in a sucrose monoalkanoate system. Langmuir, 1997, 13, 2266-2270.

[17] Pes, M. A.; Aramaki, K.; Nakamura, N.; Kunieda, H. Temperatureinsensitive microemulsionsin a sucrose monoalkanoate system $J$. Colloid Interface Sci. 1996, 178, 666-672.

[18] Fanun, M. Propylene glycol and ethoxylated surfactant effects on the phase behavior of water/sucrose stearate/oil systems. $J$. Dispers. Sci. Technol., 2007, 28, 1244-1253.

[19] Fanun, M. Conductivity, viscosity, NMR and diclofenac solubilization capacity studies of mixed nonionic surfactants microemulsions. J. Mol. Liq., 2007, 135, 5-13.

[20] Fanun, M. Microstructure of mixed nonionic surfactants microemulsions studied By SAXS and DLS. J. Dispers. Sci. Technol., 2009, 30, 115-123.
[21] Fanun, M. A Study of the Properties of Mixed Nonionic Surfactants Microemulsions by NMR, SAXS, Viscosity and Conductivity. J. Mol. Liq., 2008, 142, 103-110.

[22] Fanun, M. Properties of Microemulsions based on mixed nonionic surfactants and mixed oils. J. Mol. Liq., 2009, 150, 25-32.

[23] Fanun, M. Oil Type Effect on Diclofenac Solubilization in Mixed Nonionic Surfactants Microemulsions. Colloids \& Surfaces A: Physicochem. Eng. Asp., 2009, 343, 75-82.

[24] Fanun, M. Microemulsions with nonionic surfactants and mixed oils. Soft Mater., 2009, 7, 258-276.

[25] Treptow, R.S. Research and Development Report 1, The Procter and Gamble Company, Cinicinnati, OH, USA, 1971.

[26] Wang, L.-H.; Wang, C.-C.; Kuo, S.-C. Vehicle and enhancer effects on human skin penetration of aminophylline from cream formulations: Evaluation in vivo. J. Cos. Sci., 2007, 58, 245-254.

[27] Rickley, R.G. Solubilizing excipients in oral and injectable formulations. Pharma. Res., 2004, 21, 201-30.

[28] Glatter, O.; Strey, R.; Schubert, K.-V.; Kaler, E. W. Small angle scattering applied to microemulsions. Ber. Bunsenges. Phys. Chem., 1996, 100, 323-335.

[29] Koppel, D.E. Analysis of macro molecular polydispersity in intensity correlation spectroscopy: the method of cumulants. J. Chem. Phys., 1972, 57, 4814-4820.

[30] Provencher, S.W. Inverse problems in polymer characterisation: direct analysis of polydispersity with photon correlation spectroscopy. Macromol. Chem., 1979, 180, 201-209.

[31] Lake, J.A. An iterative method of slit-correcting small angle X-ray data. Acta Crystallogr., 1967, 23,191-194.

[32] Teubner, M.; Strey, R. Origin of the scattering peak in microemulsions J. Chem. Phys., 1987, 87, 3195-3200.

[33] Teukolsky, S.A.; Vetterling, W.T.; Flannery, B.P. Numerical Recipes in C: The Art of Scientific Computing, $2^{\text {nd }}$ ed., Cambridge University Press, New York, 1992, pp. 683-688.

[34] Billman, J.F.; Kaler, E.W. Structure and Phase Behavior in Four-Component Nonionic Microemulsions. Langmuir, 1991, 7, 1609- 1617.

[35] Fanun, M. Conductive flow parameters of mixed nonionic surfactants microemulsions. J. Disp. Sci. Technol., 2008, 29, 1426-1434.

[36] Fanun, M. Viscous flow parameters of mixed nonionic surfactants microemulsions. J. Disp. Sci. Technol., 2008, 29, 1257-1265.

[37] Gradzielski, M. Hoffmann H., In: Kumar P., Mittal K.L. Eds.: In: Handbook of Microemulsion Science and Technology, Marcel Dekker, NY, 1999, pp. 357-386.

[38] Fanun, M. Structure probing of water/mixed nonionic surfactants/caprylic-capric triglyceride system using conductivity and NMR. J. Mol. Liq., 2007, 133, 22-27.

[39] Iampietro, D. I.; Brasher, L. L.; Kaler, E. W.; Stradner, A.; Glatter, O. Direct Analysis of SANS and SAXS Measurements of Catanionic Surfactant Mixtures by Fourier Transformation. J. Phys. Chem. B., 1998, 102, 3105-3113. 